\title{
Secondary Bacterial Infections and Antimicrobial Resistance in COVID-19: Comparative Evaluation of Pre-Pandemic and Pandemic-Era, A Retrospective Single Center Study
}

\section{Mustafa Karataş}

Ege University Faculty of Medicine: Ege Universitesi Tip Fakultesi

Melike Yaşar Duman

Ege University Faculty of Medicine: Ege Universitesi Tip Fakultesi

Alper Tünger

Ege University Faculty of Medicine

Feriha Çilli

Ege University Faculty of Medicine

Şöhret Aydemir

Ege University Faculty of Medicine

Volkan Ozenci ( $\square$ volkan.ozenci@sil.se )

Karolinska Institutet https://orcid.org/0000-0002-8069-4027

\section{Original Article}

Keywords: COVID-19, secondary bacterial infections, co-infections, antimicrobial resistance

Posted Date: February 4th, 2021

DOI: https://doi.org/10.21203/rs.3.rs-157931/v1

License: (c) (i) This work is licensed under a Creative Commons Attribution 4.0 International License.

Read Full License

Version of Record: A version of this preprint was published at Annals of Clinical Microbiology and Antimicrobials on July 19th, 2021. See the published version at https://doi.org/10.1186/s12941-02100454-7. 


\section{Abstract}

Purpose: In this study, we aimed to evaluate the epidemiology and antimicrobial resistance (AMR) patterns of bacterial pathogens in COVID-19 patients and to compare the results with control groups from the pre-pandemic and pandemic era.

Methods: Microbiological database records of all the COVID-19 diagnosed patients in the Ege University Hospital between March 15, 2020, and June 15, 2020, evaluated retrospectively. Patients who acquired secondary bacterial infections (SBIs) and bacterial co-infections were analyzed. Etiology and AMR data of the bacterial infections were collected. Results were also compared to control groups from prepandemic and pandemic era data.

Results: In total, 4,859 positive culture results from 3,532 patients were analyzed. Fifty-two (3.59\%) patients had $78 \mathrm{SBIs}$ and 38 (2.62\%) patients had 45 bacterial co-infections among 1,447 COVID-19 patients. $22 / 85$ (25.88\%) patients died who had bacterial infections. The respiratory culture-positive sample rate was $39.02 \%$ among all culture-positive samples in the COVID-19 group. There was a significant decrease in extended-spectrum beta-lactamase-producing Enterobacterales (8.94\%) compared to samples from the pre-pandemic $(20.76 \%)$ and pandemic era $(20.74 \%)$ ( $p=0.001$ for both comparisons). Interestingly, Acinetobacter baumannii was the main pathogen in the respiratory infections of COVID-19 patients (\%9.76) and the rate was significantly higher than pre-pandemic $(3.49 \%, p<0.002)$ and pandemic era control groups $(3.11 \%, \mathrm{p}<0.001)$.

Conclusion: Due to the low frequency of SBls reported during the ongoing pandemic, a more careful and targeted antimicrobial prescription should be taken. While patients with COVID-19 had lower levels of ESBL producing Enterobacterales, the frequency of MDR A. baumannii is higher.

\section{Introduction}

Antimicrobial Resistance (AMR) is recognized as a public threat with constantly increasing urgency [1]. In 2050, 10 million deaths and economic loss of $\$ 100$ billion expected annually due to multidrug-resistant (resistant to more than three or more antimicrobial categories) infections [2]. It is widely accepted that antimicrobial surveillance is crucial for tackling AMR globally [3]. The current pandemic with the severe acute respiratory syndrome coronavirus 2 (SARS-CoV-2) is related to high mortality, morbidity, and healthcare-related costs [4]. Now, more than 79.2 million people have been infected and 1.7 million of them died [5]. The mortality rate has increased in patients with secondary bacterial infections (SBIs), as reported in previous studies [6]. Viral factors can also promote the production of bacterial invasive respiratory infections, such as recent pandemics ${ }^{[7-8]}$, by impairing the immune response, enhancing the destruction of cells and tissue.

In the pandemic era, deaths and hospitalizations have been reported in all age groups, with higher frequency in the elderly (> 60 years) patients [9]. SBIs has previously been reported in patients with COVID-19 $[6,11,13]$. It was recently shown that SBIs were observed in $15 \%$ of COVID-19 patients, while the 
SBIs were associated with $50 \%$ of all deaths [13]. Consequently, broad-spectrum and empirical antibiotic use become an approach for the prevent and overcome possible SBIs in these patients $[13,15,16]$. Also, hygiene procedures such as hand hygiene, usage of disinfectants, personal protective equipment (PPE) are significantly changed due to the COVID-19 pandemic [16].

In environments where personnel and personal protective equipment (PPE) are insufficient, hygienic conditions may deteriorate, as well as increased use of PPE in interventions may prevent the increase of SBIs and the spread of common resistant bacterial strains. A previous study from Italy, lack of PPE, and lack of healthcare professionals associated with increased risk of spreading the carbapenem-resistant $K$. pneumoniae in the intensive care units (ICUs) [17].

It is important to evaluate the etiology and resistance patterns of SBls as well as to compare the data with other patients before and during the pandemic. There is scarce data on SBI etiology and antimicrobial susceptibility data [18]. However, hitherto published studies have not analyzed the changes in AMR in patients with COVID-19 and controls. In addition, there is no published data from developing countries on SBls where high AMR is prevalent. The data is crucial for establishing effective antibiotic therapy as well as avoiding unnecessary treatment [19].

In this study, we aimed to evaluate the etiologies of SBIs and co-infections and AMR profiles of the bacterial pathogens causing these infections in patients with COVID-19. Also, our secondary aim was to reveal the differences in the AMR between patients with COVID-19 and other patients from the prepandemic and pandemic era.

\section{Methods}

\section{Study Design}

This study was performed at Ege University Hospital, Izmir, with a total of 2,426 patient beds. The study covers the data between December 15, 2019, and June 15, 2020, which can be considered the previous three months and the first three months of the COVID-19 pandemic in Turkey.

\section{Study Population}

COVID-19 Group: During the pandemic peak for SARS-CoV-2, real-time reverse transcription PCR (RT-PCR) assay positive and negative patients with symptoms consistent with COVID-19 were included. The RTPCR assay negative patients had radiological imaging findings meeting the Ministry of Health's probable case [20] criteria for COVID-19 and therefore included in the COVID-19 patient group [Supplementary Information]. SBIs are defined as infections occurring two days or more after patients were admitted to the hospital. Infections occurring not more than two days of hospitalization were defined as coinfections.

Control group: This study included two control groups, i.e., one pre-pandemic era group with clinical microbiology culture results registered between December 15, 2019 - March 15, 2020, and one pandemic 
era group with microbiology culture results registered between March 15, 2020 - June 15, 2020, with no clinical and/or laboratory diagnosis of COVID-19 (Fig.1).

\section{Laboratory Methods}

\section{Bacterial culture:}

The investigation of bacterial pathogens in clinical samples was conducted with standard procedures as requested by the attending physician and was evaluated by microbiologists [Supplementary Information]. Identification and antimicrobial susceptibility testing of the isolates were performed using MALDI-TOF MS (Matrix-Assisted Laser Desorption/lonization-Time of Flight Mass Spectrometry) (BioMérieux, France) and VITEK 2 (BioMérieux, France) automated systems, respectively. For viridians group streptococci, Haemophilus spp., Corynebacterium striatum, Streptococcus pneumoniae, and Stenotrophomonas maltophilia the international Kirby Bauer disk diffusion method was used. All the susceptibility results were evaluated in accordance with the EUCAST criteria [21]. When imipenem or meropenem resistance was detected in Enterobacterales strains with the automated system, the result was confirmed by gradient test (BioMérieux, France). Antibiotic MIC values were confirmed by gradient test when isolates were determined resistant to vancomycin, teicoplanin, linezolid, tigecycline with VITEK 2 automated system. The VITEK 2 cefoxitin screen test was used to detect MRSA strains. Only one isolate per patient was studied.

SARS-CoV-2 RT-PCR: For PCR tests, DirectDetect ${ }^{\mathrm{TM}}$ SARS-CoV-2 Detection Kit (Coyote Bioscience Co, China) and Bio-Speedy SARS-COV2 (2019-nCoV) qPCR Detection Kit (Bioeksen R\&D Technologies Ltd, Turkey) was used and according to the manufacturers' recommendations. The PCR tests were performed with Qiagen Rotorgene Q-5 Plex-HRM Thermal Cycler (Qiagen, Belgium).

\section{Data collection}

Blood, respiratory tract, urinary tract, and other samples such as gastrointestinal tract, tissue, normally sterile fluids cultures sent from patients were evaluated and susceptibility test results were collected from the microbiology laboratory database.

\section{Statistical analysis}

Statistical analyses were performed using Microsoft Excel 2016 and IBM SPSS Statistics Version 18.0 (IBM Corp, Armonk, NY). Cross tables were created for categorical variables and chi-square analysis was performed. Categorical variables were shown as numbers and \%, numerical variables as median (min., max.). A. baumannii changes between different groups were analyzed with two-tailed Fisher's exact test. Other comparisons (e.g., etiological changes, multidrug-resistant bacteria changes) were done with twotailed Pearson's chi-squared test. A p value $<0.05$ was considered statistically significant.

\section{Ethical approval}


This study has been approved by the Ege University Medical Research Ethics Committee (20 - 9T / 75) and the Turkish Ministry of Health.

\section{Results}

In total, 4,859 culture-positive samples from 3,532 patients were studied. The samples were collected between December 15, 2019, and June 15, 2020. The pre-pandemic era control group consists of 3,034 samples from 2,143 patients and the pandemic era control group included 1,702 samples from 1,304 patients. 1,447 COVID-19 diagnosed patients' data evaluated separately and 85 of them had 123 bacterial infections.

Of the 2,143 patients in the pre-pandemic group, 1,057 (49.32\%) were female, 1,086 (50.68\%) were male, and the median age of these patients was 52 (0-99 years). Of the 1,304 patients in the pandemic group, $630(48.31 \%)$ were female, 674 (51.69\%) were male, and the median age of these patients was $55(0-100$ years). Of 85 patients evaluated as COVID-19 patients, 45 (52.94\%) were female and 40 (47.06\%) were male and the median age of the patients was 61 (9 months-99 years). The mortality rate was \%25.88 in COVID-19 patients who had been diagnosed with bacterial infections and 64 (38-96 years) was the median age among deaths.

\section{Pre-Pandemic Control Group}

During the three months before the pandemic (December 15, 2019 - March 15, 2020), microbiological data from 2,143 patients were evaluated, and 3,034 culture-positive samples were detected. Mostly, strains were obtained from urine $(1,472,48.52 \%)$, respiratory tract samples (e.g., sputum, bronchoalveolar lavage) $(540,17.32 \%)$. The most common strains were E. coli $(766,23.55 \%)$, K. pneumoniae (324, $11.56 \%)$, and Pseudomonas aeruginosa (254, 8.37\%) (Fig 2.). ESBL producing Enterobacterales were the most common $(630,20.76 \%)$ MDR bacteria among the strains isolated from these samples.

\section{Pandemic Era Control Group}

Microbiological data from 1,304 patients were evaluated and 1,702 culture-positive samples were included in the pandemic era control group. Strains were obtained from urine $(796,46.77 \%)$, blood (276, $16.22 \%)$, lower respiratory tract samples (261, 15.33\%), and others (e.g., feces, tissue, and sterile fluid). The most common isolated strains were E. coli $(447,26.26 \%)$, K. pneumoniae $(197,11.57 \%)$, and Pseudomonas aeruginosa (136, 7.99\%) (Table 1.). ESBL producing Enterobacterales were the most common $(353,20.74 \%)$ type of MDR strain, followed by Carbapenem-resistant Enterobacterales (CRE) $(62,3.64 \%)$.

Table 1 Bacterial strains detected in patients with COVID-19 and controls 
Control Group

Group

Patients

\begin{tabular}{|c|c|c|c|c|c|c|}
\hline & $\mathbf{N}^{\#}$ & $\%$ & $\mathbf{N}$ & $\%$ & $\mathbf{N}$ & $\%$ \\
\hline Escherichia coli & 766 & $25,25 \%$ & 447 & $26,26 \% *$ & 22 & $17,89 \% *$ \\
\hline $\begin{array}{l}\text { Klebsiella } \\
\text { pneumoniae }\end{array}$ & 324 & $10,68 \%$ & 197 & $11,57 \%$ & 15 & $12,20 \%$ \\
\hline $\begin{array}{l}\text { Acinetobacter } \\
\text { baumannii }\end{array}$ & 106 & $3,49 \% * * *$ & 53 & $3,11 \% * * *$ & 12 & $9,76 \% * * *$ \\
\hline $\begin{array}{l}\text { Corynebacterium } \\
\text { striatum }\end{array}$ & 84 & $2,77 \%$ & 39 & $2,29 \%$ & 5 & $4,07 \%$ \\
\hline $\begin{array}{l}\text { Enterococcus } \\
\text { faecium }\end{array}$ & 174 & $5,74 \%$ & 115 & $6,76 \%$ & 8 & $6,50 \%$ \\
\hline $\begin{array}{l}\text { Enterococcus } \\
\text { faecalis }\end{array}$ & 201 & $6,62 \%$ & 122 & $7,17 \%$ & 9 & $7,32 \%$ \\
\hline $\begin{array}{l}\text { Pseudomonas } \\
\text { aeruginosa }\end{array}$ & 254 & $8,37 \%$ & 136 & $7,99 \%$ & 8 & $6,50 \%$ \\
\hline $\begin{array}{l}\text { Staphylococcus } \\
\text { aureus }\end{array}$ & 193 & $6,36 \%$ & 112 & $6,58 \%$ & 11 & $8,94 \%$ \\
\hline Proteus mirabilis & 79 & $2,60 \%$ & 37 & $2,17 \%$ & 3 & $2,44 \%$ \\
\hline Other & 853 & $28,11 \%$ & 444 & $26,09 \%$ & 30 & $24,39 \%$ \\
\hline Total & 3,034 & $100,00 \%$ & 1,702 & $100,00 \%$ & 123 & $100,00 \%$ \\
\hline
\end{tabular}

${ }^{\#} \mathrm{~N}=$ numbers

$* \mathrm{p}<0.05$

$* * * p=0.001$

\section{COVID-19 Patients}

The existence of bacterial infections in 1,447 COVID-19 patients (both inpatient and outpatient) were evaluated. In total, 52/1,447 (3.59\%) patients had SBIs and 38/1,447 (\%2.62) had bacterial co-infections. Bacterial isolates were detected in 123 clinical samples. A seconder bacterial infection developed in five patients with bacterial co-infection during their follow-up. 24 (28.24\%) patients had multiple bacterial, and 
$61(71.76 \%)$ patients had mono-bacterial infections. The most common MDR organisms were ESBL producing Enterobacterales (11, 8.94\%) (Fig 3.).

In total, 78/123 (63.41\%) bacterial strains were obtained two days after when the patients were hospitalized and described as hospital-acquired infections while the 45/123 (36.59\%) were communityacquired infections. The most common bacteria was $A$. baumannii $(10,9.76 \%)$ among all respiratory tract samples (Table 2.).

Table 2 Strains isolated from COVID-19 patients

\begin{tabular}{lrrrr|r} 
& UTI & RTI & BSI & Other & Total \\
\hline Klebsiella pneumoniae & 9 & 4 & 2 & & 15 \\
Enterococcus faecalis & 8 & & 1 & & 9 \\
Escherichia coli & 18 & 1 & 3 & & 22 \\
Acinetobacter baumannii & 2 & 10 & & & 12 \\
\hline Enterococcus faecium & 8 & & & & 8 \\
\hline Staphylococcus aureus & 1 & 7 & 1 & 2 & 11 \\
\hline Pseudomonas aeruginosa & 2 & 5 & & 1 & 8 \\
\hline Corynebacterium striatum & & 5 & & & 5 \\
\hline Other & 8 & 16 & 5 & 4 & 33 \\
\hline Total & $\mathbf{5 6}$ & $\mathbf{4 8}$ & $\mathbf{1 2}$ & $\mathbf{7}$ & $\mathbf{1 2 3}$
\end{tabular}

UTI: Urinary Tract Infections RTI: Respiratory Tract Infections BSI: Bloodstream Infections Other: Feces, tissue, and sterile fluid

\section{Discussion}

Viral respiratory infections have been associated with an increased risk of bacterial infections. During the $2009 \mathrm{H} 1 \mathrm{~N} 1$ outbreak, within 72 hours of intensive care unit (ICU) admission, $30.3 \%$ of cases had bacterial co-infection [22]. Pandemics and seasonal flu data suggest that bacterial infections can worsen viral diseases and causes severe outcomes. Patients receiving invasive mechanical ventilation in other SARS and MERS epidemics developed secondary infections and had higher mortality [23].

In the present study, $2.62 \%$ of patients with COVID-19 had bacterial co-infections whereas $3.59 \%$ had secondary bacterial infections. In total, 22/85 (25.88\%) patients with bacterial infections died. UTI was 
the most common infection type (45.5\%) and followed by RTI (39.02\%) which was significantly higher than the two control groups. Previous studies analysing SBI in patients with COVID-19 are contradictory. He et al showed that $50 \%$ of the patients with COVID-19 had a seconder bacterial infection or carried bacterial pathogens [24]. However, in a recent meta-analysis, it was reported that the SBI rate in COVID-19 patients was between $4.7-19.5 \%$ and was associated with an increased risk of severe course or fatal outcomes [26-27]. The underlying reason for low bacterial infection rates in the present study is not known. It might be related to several factors including the severity of the disease in patients included prior antimicrobial therapy or stringent local hygiene protocols applied during the pandemic era [27]. The observation of $3.59 \%$ of $\mathrm{SBI}$ indicates that it is necessary to be restrictive in empiric antibiotic treatment of patients with COVID-19.

The most common pathogens in the COVID-19 study group were E. coli $(22,17.89 \%)$, K. pneumonieae (15, $12.2 \%)$, A. baumannii $(12,9.76 \%)$, and S. aureus $(11,8.94 \%)$. Detection of $E$. coli $(22,17.89 \%)$ was significantly lower in patients with COVID-19, compared to the pandemic era control group $(447,26.26 \%)$ $(p=0.04)$. In contrast, detection of $A$. baumannii in patients with COVID-19 was higher than in the two control groups.

In lower respiratory tract infections, the most detected pathogens were Gram-negative bacteria (32, $66.66 \%)$, following by Gram-positive bacteria (16,33.33\%). In Gram-negative bacteria, the most common isolated strains were $A$. baumannii (10/32, 31.25\%), followed by $P$. aeruginosa (5/32, 15.63\%). Among Gram-positive bacteria, $S$. aureus $(7 / 16,43.75 \%)$ was the most common isolated strain. Although Gramnegative and Gram-positive bacteria distribution were similar, there was a noteworthy higher $A$. baumannii occurrence in our COVID-19 study group and needs further evaluation with comprehensive clinical data. A recent study from China showed that the most common bacterial pathogens isolated from respiratory tract samples were Gram-negative bacteria $(26,65 \%)$, following by Gram-positive bacteria $(14,34.99 \%)$. In that study, the most common bacterial pathogens encompassed $K$. pneumoniae $(\mathrm{n}=11)$, E. faecium $(\mathrm{n}=9)$, and followed A. baumannii $(\mathrm{n}=8)$ [6]. The underlying reason for discrepant results between the present study and the previous report is not known.

Extended-spectrum beta-lactamase-producing Enterobacterales were significantly lower in COVID-19 compared to pre-pandemic $(p=0.001)$ and pandemic era control group $(p=0.002)$. In a recent study which is reported from Egypt, Gram-negative isolates were mostly ESBL or carbapenemase producers which differs from our study [28]. The distinction may be due to the difference in the drug resistance profile between countries and/or local hygiene measures taken for patients with COVID-19. MDR $A$. baumannii was the most common bacteria $(9.76 \%)$ in respiratory tract samples (39.02\%) in COVID-19 patients and significantly more than pre-pandemic $(3.49 \%)(p=0.002)$ and pandemic $(3.11 \%)(p=0.001)$ era. In another study from New Jersey, when COVID-19 cases surge, increased carbapenem-resistant Acinetobacter baumannii (CRAB) counts were reported. CRABs were mostly among COVID-19 patients admitted to ICUs and receiving ventilation therapy [29]. In both cases, the increase in intervention needed for COVID-19 patients may have led to a deterioration in hygiene conditions and an increase in the spread of MDR A. baumannii. 
The present study has some limitations. First, not all COVID-19 patients were confirmed by PCR and the comprehensive clinical data and disease severity of these patients were not studied. Secondly, a limited number of patients available in our single-center study. However, we followed the guidelines in the diagnosis of COVID-19, and it is highly unlikely that the patients with typical COVID-19 radiological findings had other infections. The lack of clinical severity data might be important, but the study focuses on the description of the prevalence of microorganisms and AST results in patients with COVID-19 in general. In addition, the sample size covers a period of 3 months in the pandemic era. The study has the following strengths: First, unlike other studies, we compared both the prevalence of bacterial infections and antimicrobial resistance patterns in patients with COVID-19 and control groups. Also, this study is the first comparative study presented from Turkey which is a developing country with a general high MDR bacteria profile.

\section{Conclusion}

In conclusion, the study shows that SBIs and bacterial coinfections were low in COVID-19 patients; but when it occurs, it causes severe outcomes and related to mortality. According to these, empirical broad spectrum antimicrobial prescribing should be evaluated more carefully. Also, when COVID-19 patients had a lower risk of infected with ESBL producing Enterobacterales; an increase in the MDR A. baumannii rates and increase of respiratory tract samples is remarkable and needs further evaluation together. This is also important for diagnosis, possible treatments, and evaluating the sanitation measures in the hospitals. Further studies with a larger sample size and detailed clinical data are warranted.

\section{Declarations}

\section{Acknowledgements}

\section{Declarations}

Funding: The author(s) received no specific funding for this work.

Conflicts of interest/Competing interests: Authors declare no competing interests.

Availability of data and material: Authors can confirm that all relevant data are included in the article and/or its supplementary information files.

Code availability: Not applicable.

Authors' contributions: Not applicable.

Ethics approval: This study has been approved by the Ege University Medical Research Ethics Committee (20 - 9T / 75). 
Consent to participate: Not applicable.

Consent for publication: Not applicable.

\section{References}

[1] Nieuwlaat R et al (2020) Coronavirus Disease 2019 and Antimicrobial Resistance: Parallel and Interacting Health Emergencies. Clin. Infect. Dis. doi: 10.1093/cid/ciaa773.

[2] O ' Neill J (2014) Antimicrobial Resistance: Tackling a crisis for the health and wealth of nations The Review on Antimicrobial Resistance Chaired no. December, 2014.

[3] Ahmad M, Khan AU. Global economic impact of antibiotic resistance: A review. J Glob Antimicrob Resist. 2019 Dec;19:313-316. doi: 10.1016/j.jgar.2019.05.024. Epub 2019 Jun 5. PMID: 31176071.

[4] Sher L. The impact of the COVID-19 pandemic on suicide rates. QJM. 2020 Oct 1;113(10):707-712. doi: 10.1093/qjmed/hcaa202. PMID: 32539153; PMCID: PMC7313777.

[5] WHO. (OMS), COVID-19 Weekly Epidemiological Update no. November, p. 1;4, 2020, [Online]. Available: https://www.who.int/docs/default-source/coronaviruse/situation-reports/20201012-weeklyepi-update-9.pdf

[6] Zhang $\mathrm{H}$, Zhang $\mathrm{Y}, \mathrm{Wu} \mathrm{J}$ et al. Risks and features of secondary infections in severe and critical ill COVID-19 patients. Emerg Microbes Infect. 2020 Dec;9(1):1958-1964. doi:

10.1080/22221751.2020.1812437. PMID: 32815458.

[7] Morris DE, Cleary DW, Clarke SC. Secondary Bacterial Infections Associated with Influenza Pandemics. Front Microbiol. 2017 Jun 23;8:1041. doi: 10.3389/fmicb.2017.01041. PMID: 28690590; PMCID: PMC5481322.

[8] Liderot $\mathrm{K}$, Ahl M, Ozenci V. Secondary bacterial infections in patients with seasonal influenza A and pandemic H1N1. Biomed Res Int. 2013;2013:376219. doi: 10.1155/2013/376219. Epub 2013 Jun 20. PMID: 23865050; PMCID: PMC3705841.

[9] Li X, Xu S, Yu M et al. Risk factors for severity and mortality in adult COVID-19 inpatients in Wuhan. J Allergy Clin Immunol. 2020 Jul;146(1):110-118. doi: 10.1016/j.jaci.2020.04.006. Epub 2020 Apr 12. PMID: 32294485; PMCID: PMC7152876.

[10] $\mathrm{Li} \mathrm{J}$ et al. Etiology and antimicrobial resistance of secondary bacterial infections in patients hospitalized with COVID-19 in Wuhan, China: a retrospective analysis. Antimicrobial Resistance and Infection Control (2020) 9:153. doi: 10.1186/s13756-020-00819-1.

[11] Huang C, Wang Y, Li X et al. Clinical features of patients infected with 2019 novel coronavirus in Wuhan, China. Lancet. 2020 Feb 15;395(10223):497-506. doi: 10.1016/S0140-6736(20)30183-5. Epub 
2020 Jan 24. Erratum in: Lancet. 2020 Jan 30;: PMID: 31986264; PMCID: PMC7159299.

[12] Zhou F, Yu T, Du R et al. Clinical course and risk factors for mortality of adult inpatients with COVID19 in Wuhan, China: a retrospective cohort study. Lancet. 2020 Mar 28;395(10229):1054-1062. doi: 10.1016/S0140-6736(20)30566-3. Epub 2020 Mar 11. Erratum in: Lancet. 2020 Mar 28;395(10229):1038. Erratum in: Lancet. 2020 Mar 28;395(10229):1038. PMID: 32171076; PMCID: PMC7270627.

[13] Yang $X$ et al (2020) Clinical course and outcomes of critically ill patients with SARS-CoV-2 pneumonia in Wuhan, China: a single-centered, retrospective, observational study. Lancet Respir Med. 2020 05; 8(5):475-481. doi: 10.1016/S2213-2600(20)30079-5.

[14] RotheK, Feihl S, Schneider J et al. (2020) Rates of bacterial co-infections and antimicrobial use in COVID-19 patients: a retrospective cohort study in light of antibiotic stewardship. Eur J Clin Microbiol Infect Dis. https://doi.org/10.1007/s10096-020-04063-8.

[15] Sivapalan P, Ulrik CS, Bojesen RD et al. (2020) Proactive Prophylaxis With Azithromycin and HydroxyChloroquine in Hospitalised Patients With COVID-19 (ProPAC-COVID): A structured summary of a study protocol for a randomised controlled trial. Trials. 21(1):513. doi: 10.1186/s13063-020-04409-9..

[16] Metin N, Turan Ç, Utlu Z. (2020) Changes in dermatological complaints among healthcare professionals during the COVID-19 outbreak in Turkey. Acta Dermatovenerol Alp Pannonica Adriat. 29(3):115-122. PMID: 32975297.

[17] Tiri B, Sensi E, Marsiliani V, et al. (2020) Antimicrobial Stewardship Program, COVID-19, and Infection Control: Spread of Carbapenem-Resistant Klebsiella Pneumoniae Colonization in ICU COVID-19 Patients. What Did Not Work? J Clin Med. 2020;9(9):2744. doi:10.3390/jcm9092744

[18] Rochtus A, Izzi B, Vangeel E, et al. (2015) DNA methylation analysis of Homeobox genes implicates HOXB7 hypomethylation as risk factor for neural tube defects. Epigenetics. 2015;10(1):92-101. doi:10.1080/15592294.2014.998531

[19] Morens DM, Taubenberger JK, Fauci AS. (2008) Predominant role of bacterial pneumonia as a cause of death in pandemic influenza: implications for pandemic influenza preparedness. J Infect Dis. 2008;198(7):962-970. doi:10.1086/591708

[20] The Republic of Turkey Ministry of Health, 'COVID-19 (SARS-COV-2 Infection) (Study of Scientific Board) General Information, Epidemiology and Diagnosis', vol. 19, no. May, pp. 1-21, 2020.

[21] 'The European Committee on Antimicrobial Susceptibility Testing. Breakpoint tables for interpretation of MICs and zone diameters. Version 9.0', 2019. http://www.eucast.org.

[22] Rice TW, Rubinson L, Uyeki TM et al., (2012) NHLBI ARDS Network. Critical illness from 2009 pandemic influenza A virus and bacterial coinfection in the United States. Crit Care Med. 2012 May;40(5):1487-98. doi: 10.1097/CCM.0b013e3182416f23. 
[23] Yap FH, Gomersall CD, Fung KS et al. (2004) Increase in methicillin-resistant Staphylococcus aureus acquisition rate and change in pathogen pattern associated with an outbreak of severe acute respiratory syndrome. Clin Infect Dis. 2004 Aug 15;39(4):511-6. doi: 10.1086/422641.

[24] He F, Xia X, Nie D et al. (2020) Respiratory bacterial pathogen spectrum among COVID-19 infected and non-COVID-19 virus infected pneumonia patients. Diagn Microbiol Infect Dis. 2020 Dec;98(4):115199. doi: 10.1016/j.diagmicrobio.2020.115199.

[25] Vazzana N, Dipaola F, Ognibene S. (2020) Procalcitonin and secondary bacterial infections in COVID-19: association with disease severity and outcomes. Acta Clin Belg. 2020 Sep 23:1-5. doi: 10.1080/17843286.2020.1824749.

[26] Langford BJ, So M, Raybardhan S et al. (2020) Bacterial co-infection and secondary infection in patients with COVID-19: a living rapid review and meta-analysis. Clin Microbiol Infect. 2020 Dec;26(12):1622-1629. doi: 10.1016/j.cmi.2020.07.016.

[27] Kodaz H. (2020) Successful Treatment Strategy of Turkey against Covid-19 Outbreak. Eurasian J. Med. Oncol., 2020, doi: 10.14744/ejmo.2020.12345.

[28] Ramadan HK, Mahmoud MA, Aburahma MZ et al. (2020) Predictors of Severity and Co-Infection Resistance Profile in COVID-19 Patients: First Report from Upper Egypt. Infect Drug Resist. 2020 Oct 5;13:3409-3422. doi: 10.2147/IDR.S272605.

[29] Perez S et al. (2020) Increase in Hospital-Acquired Carbapenem-Resistant Acinetobacter baumannii Infection and Colonization in an Acute Care Hospital During a Surge in COVID-19 Admissions - New Jersey, February-July 2020. MMWR. Morb. Mortal. Wkly. Rep., vol. 69, no. 48, p. 1827, Dec. 2020, doi: 10.15585/mmwr.mm6948e1.

\section{Figures}




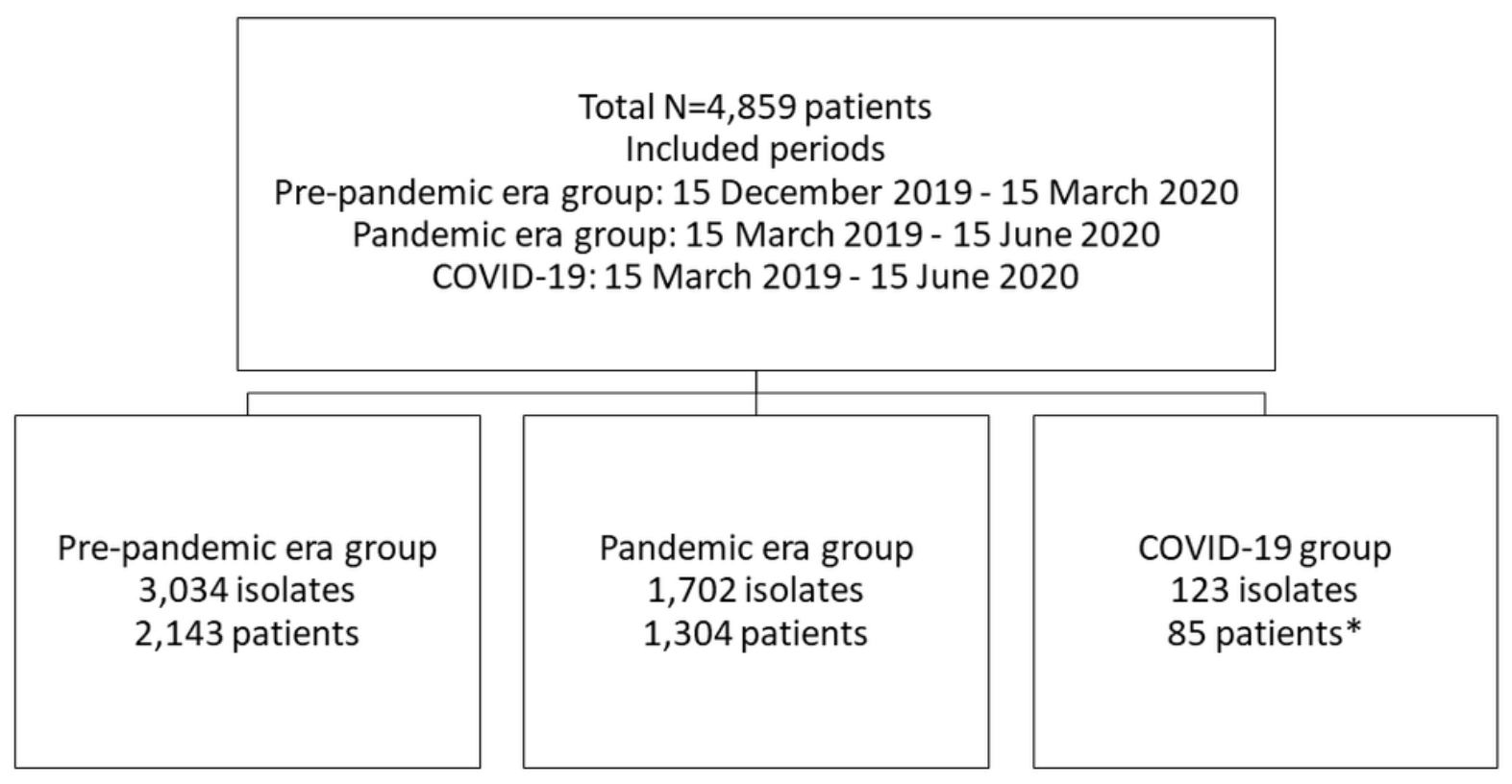

\section{Figure 1}

Study Diagram. A flowchart. There are 4 boxes, one above and the other 3 boxes below and linked to it. What is written in the upper box: 4,859 isolates and 3,532 patients' microbiological data included to study and evaluated in three separate groups. Before the pandemic: 15.12.2019-15.03.2020. Pandemic era: 15.03.2020 - 15.06.2020. COVID-19: 15.03.2020 - 15.06.2020. In the first box below: Before the Pandemic, 3,034 isolates and 2,143 patients found in microbiological database. In the second box below: Pandemic Era, 1,702 isolates and 1,304 patients found in microbiological database. In the third box below: COVID19,123 isolates and 85 patients included to study after evaluation of 1,447 COVID-19 patients' data. 


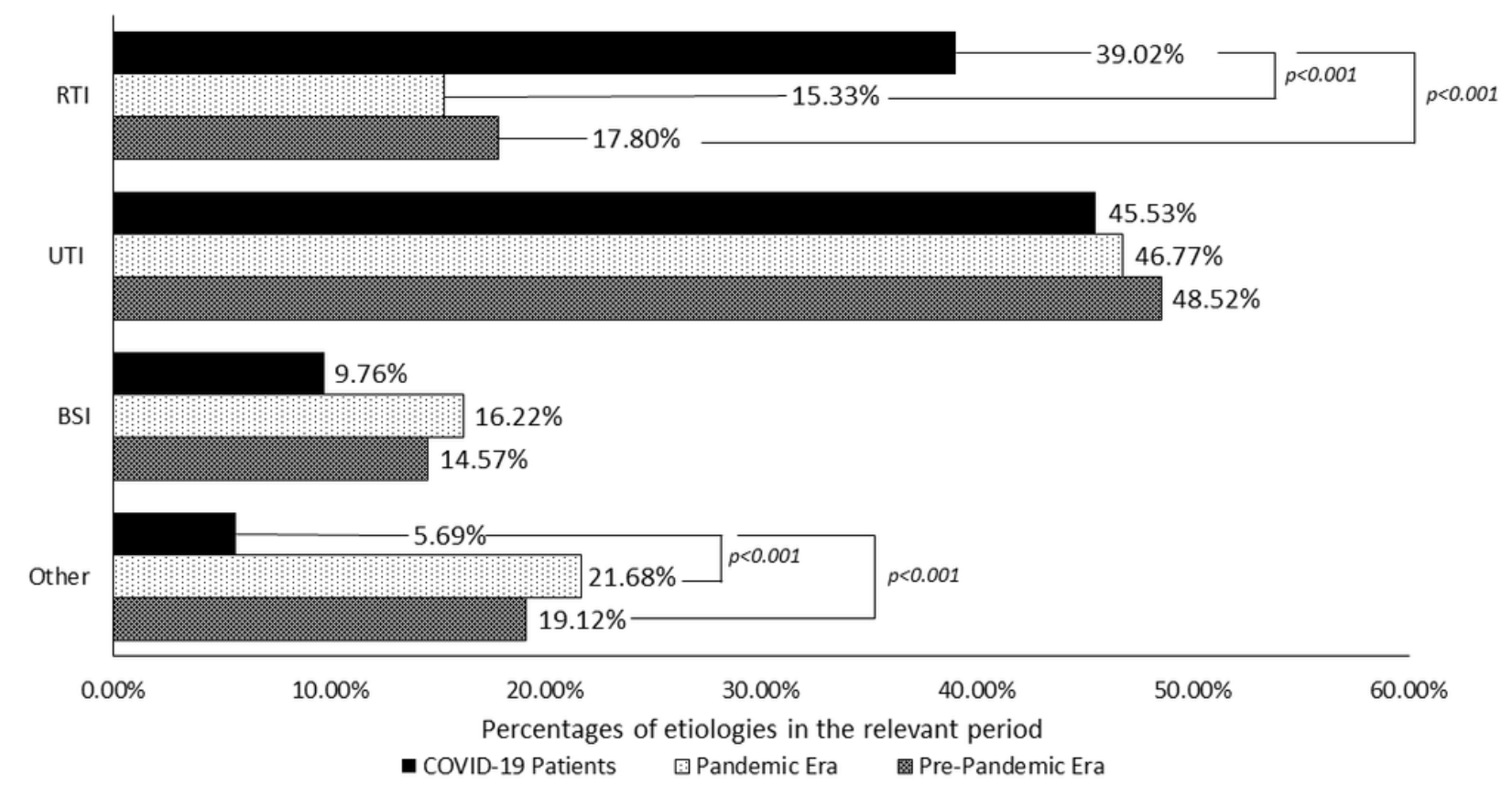

Figure 2

Etiology of the bacterial infections. A rod graph which describes etiologies of bacterial infections in different groups as percentages. $X$ axis shows percentages, y axis describes infection types such as respiratory tract infections (RTI), urinary tract infection (UTI), bloodstream infection (BSI), other (i.e., feces, sterile fluids, tissue), respectively. Every group has 3 rods in the graph. In the "RTI"; COVID-19 patients', during the pandemic, before pandemic percentages are $39.02 \%, 15.33 \%$, and $17.80 \%$, respectively. In the "UTI"; COVID-19 patients', during the pandemic, before pandemic percentages are $45.53 \%, 46.77 \%$, and $48.52 \%$, respectively. In the "BSI"; COVID-19 patients', during the pandemic, before pandemic percentages are $9.76 \%, 16.22 \%$, and $14.57 \%$, respectively. In the "Other"; COVID-19 patients', during the pandemic, before pandemic percentages are $5.69 \%, 21.68 \%$, and $19.12 \%$, respectively. 


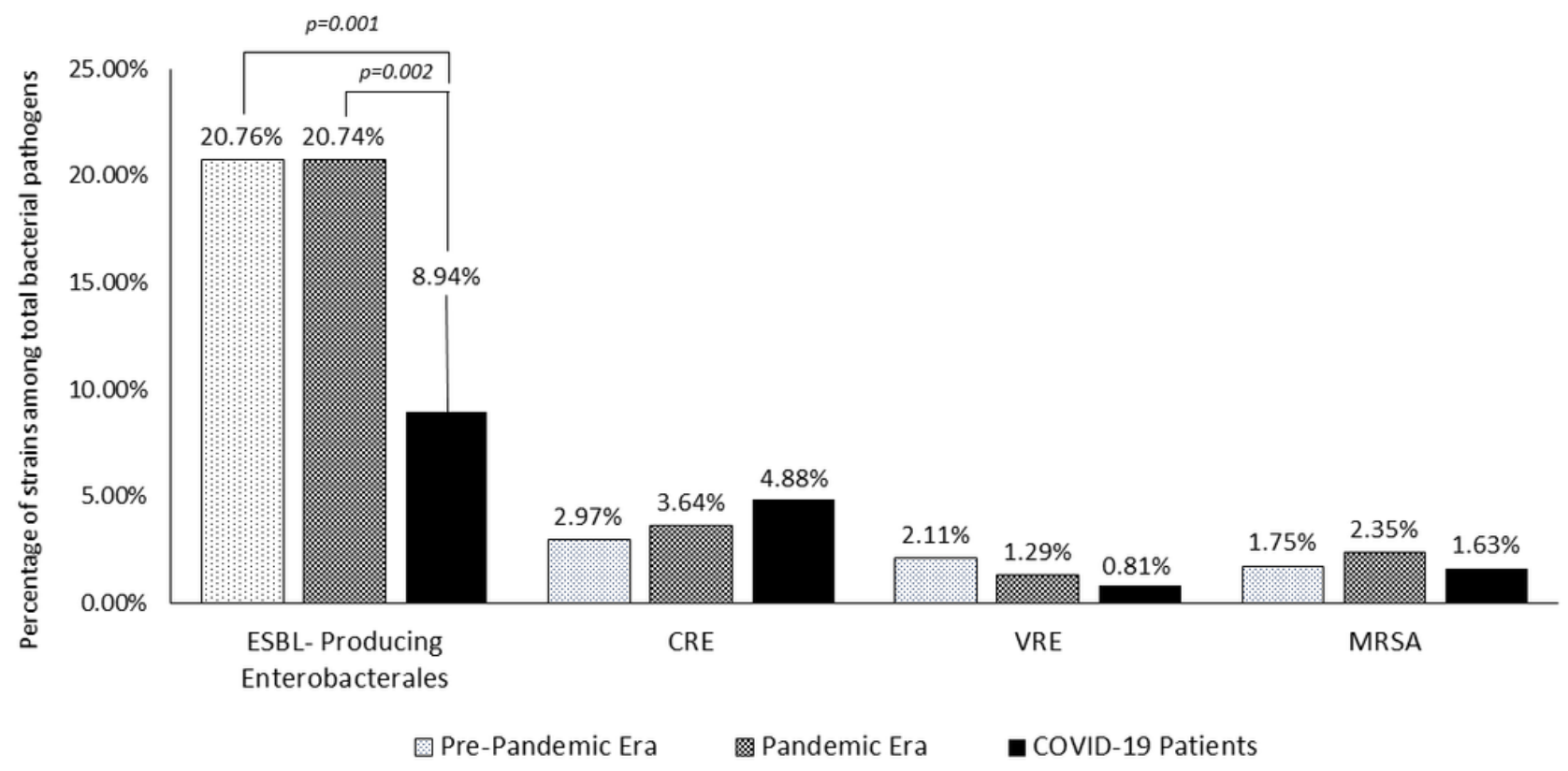

\section{Figure 3}

Common Multi Drug-Resistant Isolates. A bar graph which describes common multi drug-resistant isolates and their percentages in different groups. $X$ axis is multi drug-resistant bacteria types such as ESBL-producing Enterobacterales, Carbapenem-resistant Enterobacterales (CRE), Vancomycin-resistant Enterococci (VRE), Methicillin-resistant Staphylococcus aureus (MRSA), respectively. Y axis shows percentages. In the "BSI"; COVID-19 patients', during the pandemic, before pandemic percentages are $9.76 \%, 16.22 \%$, and $14.57 \%$, respectively. In the "ESBL"; before pandemic, during the pandemic, COVID-19 patients' percentages are $20.76 \%, 20.74 \%$, and $8.94 \%$, respectively. In the "CRE"; before pandemic, during the pandemic, COVID-19 patients' percentages are $2.97 \%, 3.64 \%$, and $4.88 \%$, respectively. In the "VRE"; before pandemic, during the pandemic, COVID-19 patients' percentages are $2.11 \%, 1.29 \%$, and $0.81 \%$, respectively. In the "MRSA"; before pandemic, during the pandemic, COVID-19 patients' percentages are $1.75 \%, 2.35 \%$, and $1.63 \%$, respectively.

\section{Supplementary Files}

This is a list of supplementary files associated with this preprint. Click to download.

- SupplementaryInformation1.docx

- Supplementarylnformation2.docx 